\title{
Implications of the complete basis set limit in valence bond theory: a case study of molecular hydrogen
}

\author{
Brian J. Duke $^{1} \cdot$ Remco W. A. Havenith ${ }^{2,3}$
}

Received: 10 November 2015 / Accepted: 3 February 2016 / Published online: 5 March 2016

(C) The Author(s) 2016. This article is published with open access at Springerlink.com

\begin{abstract}
The use of larger basis sets to approach the complete basis limit, now common in quantum chemistry, is applied for the first time to a range of valence bond functions for the simplest case of molecular hydrogen. Good convergence of the energy is slow due to difficulty in getting a correct cusp near the nuclei, but it is significant. The form of the orbitals converges much faster, leading to a slight distortion of the valence bond orbitals and an enhanced overlap, irrespective whether the basis set is restricted to basis functions centred on one atom for each valence bond orbital or the full use of the basis set is allowed. This blurs the distinction between these two approaches and shows that basis set restrictions are not tenable in the complete basis set limit. Furthermore, it supports the general use of the full basis as advocated in the spin-coupled and generalised valence bond methods.
\end{abstract}

Keywords Valence bond theory - Complete basis set limit $\cdot$ Hydrogen molecule $\cdot$ Breathing orbitals $\cdot$ Strictly localised orbitals $\cdot$ Overlap enhanced (delocal) orbitals

Remco W. A. Havenith

r.w.a.havenith@rug.nl

1 Monash Institute of Pharmaceutical Sciences, Monash University, 381 Royal Parade, Parkville, VIC 3052, Australia

2 Theoretical Chemistry, Zernike Institute for Advanced Materials and Stratingh Institute for Chemistry, University of Groningen, Nijenborgh 4, 9747 AG Groningen, The Netherlands

3 Ghent Quantum Chemistry Group, Department of Inorganic and Physical Chemistry, Ghent University, Krijgslaan 281 (S3), 9000 Ghent, Belgium

\section{Introduction}

Valence bond (VB) theory is an old theory, going back to the first quantum mechanics study of the hydrogen molecule by Heitler and London [1], which was followed by many other studies listed by McLean et al. [2]. It continues to have relevance in the way we think about molecules and the covalent bond, and, in the guise of various methods that are often called "modern valence bond" theory, it is proving a useful computational method. The concept of the complete basis set (CBS) limit is also an old one, with the Hartree-Fock limit $[3,4]$ introduced early in the development of the Hartree-Fock method. More recently, it has become common to use extrapolation formula for results obtained from a systematically chosen series of basis sets to estimate the energy at this limit [5]. Sometimes different extrapolation formulas are used for the Hartree-Fock energy and the correlation energy $[6,7]$.

The CBS limit can be reached by many different choices of basis set. It is unique, and it removes the uncertainty in the choice of the basis set. The idea of a CBS limit is that if enough functions in a given space are used, any function in that space can be expanded in that basis set of functions.

While the extrapolation method is valuable, the idea of the CBS limit is much more valuable. It underpins quantum chemical methods by showing that they are approximations which have a definite limit. The limit is independent of the choice of basis set or other means of approximating a particular wavefunction. The Hartree-Fock limit, for example, means that there is a best result for the Hartree-Fock single determinant and a set of best molecular orbitals that are used to form the single determinant. While we rarely aim to obtain that limit, or indeed need to, its presence gives 
validity to more approximate methods and also shows that orbitals that give a lower total energy are better orbitals than those in methods that give a higher energy.

VB theory is about obtaining insight and simple pictures. Such insight should not depend on the choice of basis set. Just considering the CBS limit allows us to remove the influence of the basis set, and can gain better insight into the nature of any particular method. Here we use the CBS limit to understand and gain insight into a range of valence bond methods, using the simplest system of molecular hydrogen. Although it is an exceptional system because of the lack of core electrons [8], molecular hydrogen provides illustrative insight into chemical bonding. We also use the idea that valence bond methods are related to complete active space self-consistent field (CASSCF) methods. This is implicit in the work of Cooper et al. [9] where spin-coupled (SC) [10-14] VB wavefunctions are projected out from CASSCF functions. This idea is important and allows VB methods to be grouped according to the CASSCF method that they approximate or, in some cases, are equal to.

VB theory uses one, or a linear combination of several, $n$-electron function(s). These functions are known as structures, although strictly structures are pictures of bonding that map to the $n$-electron functions. These $n$-electron functions can be constructed in various ways such as one or more Slater determinants. These Slater determinants are built from 1-electron functions, called valence bond orbitals (VBOs). The 1-electron functions are identified, in some sense, with atomic orbitals. Indeed, the very first VB calculation, the Heitler-London method, used the exact $1 \mathrm{~s}$ atomic orbital for the free hydrogen atom. For many electron molecules, solutions of the atomic Hartree-Fock equations could be used. However, this use is not satisfactory, as atomic orbitals change on molecular formation, so the use of these atomic orbitals gives rather poor energies. For the hydrogen molecule, for example, Wang [15] showed that optimising the exponent of the $1 s$ atomic orbitals gave better results. In modern VB theory, the 1-electron orbitals are chosen to be a linear combination of a set of basis functions. The coefficients of these basis functions and the coefficients of the valence bond structures are then optimised simultaneously.

Questions then arise about the choice of basis functions, both in the general sense that is common to other ab initio quantum chemistry methods, such as the size of the basis set and the choice of polarisation functions, and in a more specific VB sense. It has been argued that the basis functions for a given VBO must be restricted to those centred on a specific atom to ensure a strict local character and mapping to an atomic orbital [16]. An alternative argument is to use the full basis set, without any restriction, and then examine the VBOs to determine whether they can still be considered as atomic orbitals. In general, those resulting orbitals are reasonably localised but distorted slightly towards neighbouring atoms compared to the VBOs from the more restricted approach. Such distortions are reasonable because the atomic orbital in a molecule is now subject to the field of other nuclei. These two approaches have recently been labelled "VB-local" and "VB-delocal" [17], and these terms will be used here. The VBOs from the "VB-local" approach have been described as "strictly localised", while those from the "VB-delocal" approach have been described as "overlap enhanced orbitals" (OEOs) [18, 19]. These descriptions will be critically reviewed later in this report. "Bond distorted orbitals" (BDOs) [20] have also been introduced, which are delocalised over the two atoms in the bond. The VB-local approach is of course restricted to a basis set of atom-centred functions, while the VB-delocal approach can use a more general basis.

Using the minimum basis set approach of a single atomic orbital on each atom in the hydrogen molecule, the Heitler-London (HL) method used the two orbitals coupled together as in Eq. 1. The two atomic orbitals on atoms A and $\mathrm{B}$ are designated $\psi_{\mathrm{a}}$ and $\psi_{\mathrm{b}}$.

$\psi(\mathrm{HL})=\left[\psi_{\mathrm{a}}(1) \psi_{\mathrm{b}}(2)+\psi_{\mathrm{b}}(1) \psi_{\mathrm{a}}(2)\right][\alpha(1) \beta(2)-\beta(1) \alpha(2)]$

Ionic-covalent resonance (ICR), originally proposed by Weinbaum [21], adds ionic structures as in Eq. 2.

$$
\begin{aligned}
\psi(\mathrm{ICR})=\{ & {\left[\psi_{\mathrm{a}}(1) \psi_{\mathrm{b}}(2)+\psi_{\mathrm{b}}(1) \psi_{\mathrm{a}}(2)\right]+\lambda\left[\psi_{\mathrm{a}}(1) \psi_{\mathrm{a}}(2)\right.} \\
& \left.\left.+\psi_{\mathrm{b}}(1) \psi_{\mathrm{b}}(2)\right]\right\}[\alpha(1) \beta(2)-\beta(1) \alpha(2)]
\end{aligned}
$$

The approach by Coulson and Fischer (CF) [22] used combinations of the two orbitals in the VBOs to give a function similar to HL, as in Eq. 3.

$$
\begin{aligned}
\psi(\mathrm{CF})= & {\left[\left(\psi_{\mathrm{a}}+\lambda^{\prime} \psi_{\mathrm{b}}\right)(1)\left(\psi_{\mathrm{b}}+\lambda^{\prime} \psi_{\mathrm{a}}\right)(2)+\left(\psi_{\mathrm{b}}+\lambda^{\prime} \psi_{\mathrm{a}}\right)(1)\right.} \\
& \left.\left(\psi_{\mathrm{a}}+\lambda^{\prime} \psi_{\mathrm{b}}\right)(2)\right][\alpha(1) \beta(2)-\beta(1) \alpha(2)]
\end{aligned}
$$

The CF function is identical to the HL function, except that the latter is VB-local and the former is VB-delocal. For a minimal basis set, ICR is identical to CF, which is also equal to full configuration interaction between the two molecular orbitals of hydrogen.

In modern VB theory, we expand the VBOs (designated $\Phi_{\mathrm{A}}$ and $\Phi_{\mathrm{B}}$ for VB-delocal and $\phi_{\mathrm{A}}$ and $\phi_{\mathrm{B}}$ for VB-local) in terms of a basis set, as used in MO theory. This extends the idea of Heitler-London, ionic-covalent resonance and Coulson and Fischer by giving flexibility to the VBOs used. The extended Heitler-London wavefunction is a generalisation of the Wang wavefunction [15] and is here denoted as G-Wang:

$$
\begin{gathered}
\psi(\mathrm{G}-\text { Wang })=\left[\phi_{\mathrm{A}}(1) \phi_{\mathrm{B}}(2)+\phi_{\mathrm{B}}(1) \phi_{\mathrm{A}}(2)\right] \\
{[\alpha(1) \beta(2)-\beta(1) \alpha(2)]}
\end{gathered}
$$


Extension of the ionic-covalent resonance wavefunction corresponds to a generalisation of the Weinbaum wavefunction [21] and is here denoted as G-Weinbaum:

$$
\begin{aligned}
\psi(\mathrm{G}-\text { Weinbaum })= & \left\{\left[\phi_{\mathrm{A}}(1) \phi_{\mathrm{B}}(2)+\phi_{\mathrm{B}}(1) \phi_{\mathrm{A}}(2)\right]+\lambda\left[\phi_{\mathrm{A}}(1) \phi_{\mathrm{A}}(2)\right.\right. \\
& \left.\left.+\phi_{\mathrm{B}}(1) \phi_{\mathrm{B}}(2)\right]\right\}[\alpha(1) \beta(2)-\beta(1) \alpha(2)]
\end{aligned}
$$

Extension of the Coulson-Fischer wavefunction is equivalent to the generalised valence bond (GVB) method [23, 24] of Goddard:

$$
\begin{gathered}
\psi(\mathrm{GVB})=\left[\Phi_{\mathrm{A}}(1) \Phi_{\mathrm{B}}(2)+\Phi_{\mathrm{B}}(1) \Phi_{\mathrm{A}}(2)\right] \\
{[\alpha(1) \beta(2)-\beta(1) \alpha(2)]}
\end{gathered}
$$

It is important to note that, in modern VB theory, the optimised form of the VBOs is different in Eqs. 4, 5 and 6. This point will be discussed further later in this report. G-Wang and G-Weinbaum expand the VB orbitals using the VBlocal approach, while the GVB method uses the VB-delocal approach.

Note there is always a resemblance between the VB and MCSCF/CASSCF wavefunctions, in spite of the nonorthogonality of the VB orbitals. The GVB wavefunction is equivalent to the $\operatorname{CASSCF}(2,2)$, which in turn is equivalent to the spin-coupled function [11-14]. Methods that use additional VBOs that have a similarity to $2 s$ or $2 p$ hydrogen atomic orbitals will be related to higher levels of CASSCF, such as $\operatorname{CASSCF}(2,4)$ where two additional molecular orbitals with high $s$ character are added to those in CAS$\operatorname{SCF}(2,2), \operatorname{CASSCF}(2,6)$ where four molecular orbitals with high $p$ character are added to those in $\operatorname{CASSCF}(2,2)$, and $\operatorname{CASSCF}(2,8)$ where both sets of orbitals are added to those in $\operatorname{CASSCF}(2,2)$. Of particular interest here is the breathing orbital method [25-28], which, by using different VBOs for the ionic structures from the covalent structure in G-Weinbaum, can include more correlation for the hydrogen molecule than obtained by $\operatorname{CASSCF}(2,2)$. The best possible energy for a particular basis set is full configuration interaction (FCI), which for the hydrogen molecule is just configuration interaction with single and double excitations (CISD).

\section{Computational details}

One popular choice for systematically improving basis sets is the use of the correlation consistent basis sets of Dunning $[29,30]$, denoted cc-pVnZ, with $n=\mathrm{D}, \mathrm{T}, \mathrm{Q}, 5$ and 6 . All calculations reported here use these basis sets. Even for the hydrogen molecule the size of the basis set gets quite large. The $h$ orbitals of the cc-pV6Z basis set have been removed, due to limitations in the integral codes used here. Elimination of the $\mathrm{h}$ functions leads to very small errors (RHF: $1.7 \times 10^{-7} E_{\mathrm{H}}$; MP2: $5.5 \times 10^{-5} E_{\mathrm{H}} ;$ FCI: $2.4 \times 10^{-5} E_{\mathrm{H}}$; GVB: $2.0 \times 10^{-8} E_{\mathrm{H}}$ ).
Calculations have been performed with the Turtle VB program [31] as implemented in GAMESS-UK [32], and the VB2000 program [33, 34] incorporated into GAMESS(US) $[35,36]$. CASSCF calculations were performed with both GAMESS-UK and GAMESS(US). Some breathing orbital calculations have been performed with the XMVB program [37, 38]. The $\mathrm{H}-\mathrm{H}$ bond length was fixed at $0.74 \AA$.

\section{Results and discussion}

\subsection{CASSCF(2,2)-related methods}

The simple methods discussed earlier, G-Wang, G-Weinbaum and GVB all employ two electrons in two orbitals. G-Wang and GVB differ only in that G-Wang uses a VBlocal basis set and GVB uses a VB-delocal basis set. Since the latter is more flexible, the energy of GVB is lower than the energy of G-Wang. However, the CBS limit orbitals and energy will be identical, because either VB-local or VBdelocal can give the best possible VB orbitals at the CBS limit.

The G-Weinbaum function is only equivalent to the GVB function for minimal basis sets; for larger basis sets, the GVB wavefunction is more flexible as the use of the VB-delocal basis sets leads to a different use of the basis functions in the GVB orbitals on the two atoms. This means that the energy of G-Weinbaum cannot be lower in energy than GVB. However, G-Weinbaum is more flexible than G-Wang, so the energy of G-Wang cannot be lower than that of G-Weinbaum. For any basis set, G-Weinbaum is bounded by G-Wang above and GVB below. Since G-Wang and GVB have the same CBS limit, G-Weinbaum must also have that same CBS limit. For larger than minimal basis sets, GVB is no longer the same as full configuration interaction; however, it is equivalent to $\operatorname{CASSCF}(2,2)$ (full configuration interaction for the two electrons in two molecular orbitals, which are also fully optimised). All three methods can be labelled as belonging to the $\operatorname{CASSCF}(2,2)$ group of methods.

The results for the energies of the three methods as a function of basis set are shown in Fig. 1 as the three lowest curves. All three methods are converging to the same limit, although the use of the VB-local basis set means that G-Wang, in particular, is slow to converge. For comparison, the energy of the RHF wavefunction is also shown. The other two "s-only" curves will be discussed later.

These results can be used to examine some of the arguments that are used to distinguish, justify or label the VBlocal and VB-delocal approaches. The use of the term "overlap enhanced orbitals" (OEOs) for the VB-delocal approach can be investigated by inspecting the overlaps 


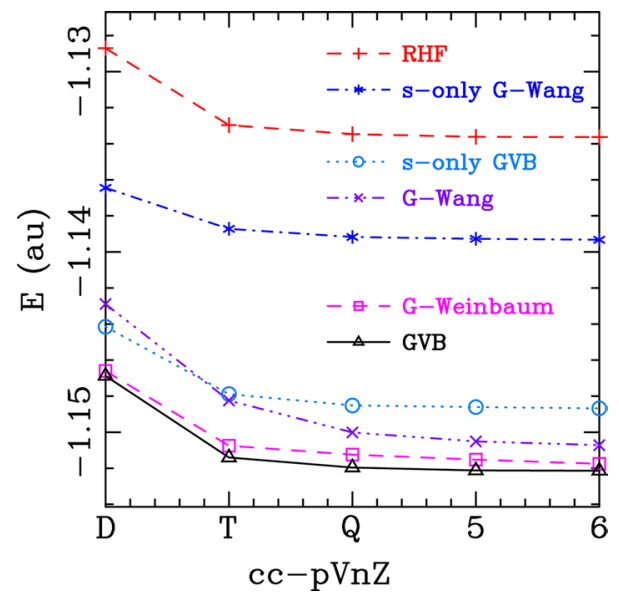

Fig. 1 RHF, s-only G-Wang, s-only GVB, G-Wang, G-Weinbaum and GVB energies as a function of basis set

(Table 1). Results for the minimal basis set, STO-6G, with optimised Slater exponents are also included as the enhancement of the overlap was first noticed with a minimal basis set. The results are striking. The overlap is certainly enhanced, but the enhancement decreases rapidly with the inclusion of higher angular momentum functions in the basis set. The $16 \%$ enhancement with a minimal basis set falls to $1 \%$ or less with the cc-pVQZ or larger basis sets. This is because the main features of the VB orbitals converge much faster than the energy converges to the CBS limit. Getting the energy to converge requires converging the wavefunction to the cusp at the nucleus. Convergence there is much slower than convergence of the orbital away from the nucleus, and it is the latter that largely determines the overlap between the two VB orbitals.

The term "strictly localised" is often used to describe the VB-local approach. While basis functions are centred at a particular atom, linear combinations used for the VBOs are not similarly centred once polarisation functions are included. The centre of the VBO can be determined by calculating the centroid of charge, relative to the atom on which the VBO is essentially localised. Another measure of localisation is the extent of the orbital along the molecular axis determined as $\left\langle Z^{2}\right\rangle$. The centroids and extents are also shown in Table 1. As with the overlaps, the difference between VB-local and VB-delocal rapidly decreases as the basis set size is increased. The VB-delocal orbitals are distorted into the bond, but the VB-local orbitals are also becoming distorted into the bond with increasing basis set size. Distortion is not solely a characteristic of VB-delocal.

Further insight can be obtained by considering the composition of the VB orbitals with the smallest cc-pVDZ basis set. This basis set has only three basis functions on each atom that contribute to the VBOs-an inner and an outer s function and a $p$ function along the molecular axis. A simple measure of the weight of each basis function is the coefficient squared divided by the sum of squares of the three (inner $s$, outer $s$ and $p$ ) coefficients for the atom A. The weight of the $p$ function is always quite small, but it is larger in G-Wang than RHF, because it is attempting to simulate the GVB orbital. The weight of the inner $s$ is generally larger than the weight of the outer $s$ orbital. In G-Wang, the weight of the outer s is slightly larger than in the GVB, as it is also doing the work of trying to fit the best function on atom $B$ by being larger on atom $A$. The GVB function is $\left(\psi_{\mathrm{a}}+\lambda \psi_{\mathrm{b}}{ }^{\prime}\right)$. In $\psi_{\mathrm{a}}$, the weight of the $\mathrm{p}$ function is almost zero- $0.01 \%$ compared to $0.26 \%$ for RHF and $0.35 \%$ for G-Wang. The weight in $\psi_{\mathrm{a}}$ of the outer $\mathrm{s}$ is much smaller than in RHF or G-Wang. For $\psi_{\mathrm{b}}{ }^{\prime}$, the function is quite different from $\psi_{\mathrm{b}}$. The weights of the inner and outer $\mathrm{s}$ functions are almost equal, and the weight of the $\mathrm{p}$ function is much larger- $4.4 \%$.

The single $p$ function in the cc-pVDZ basis set is clearly fulfilling a different role, in the G-Wang function, from its role as a polarisation function in the RHF wavefunction. Polarisation functions are designed to distort the electron density away from a nucleus in molecular orbitals that reflects the symmetry of the molecule. They clearly can also modify the electron density on the neighbouring nucleus from an orbital that does not reflect the symmetry
Table 1 Comparison of the overlap between the two VBOs in $\mathrm{H}_{2}$, movement of the centroid of charge from the nucleus (in $\AA$ ) into the bond and extent of the orbitals as measured by $\left\langle Z^{2}\right\rangle$ (in $\AA^{2}$ ), using the G-Wang (VB-local) and GVB (VB-delocal) methods

\begin{tabular}{|c|c|c|c|c|c|c|c|}
\hline \multirow[t]{2}{*}{ Basis } & \multicolumn{3}{|l|}{ Overlap } & \multicolumn{2}{|c|}{ Centroids } & \multicolumn{2}{|c|}{ Extent $\left\langle Z^{2}\right\rangle$} \\
\hline & G-Wang & GVB & $\%$ en. $^{a}$ & G-Wang & GVB & G-Wang & GVB \\
\hline STO-6G & 0.687 & 0.797 & 16.0 & 0.000 & 0.067 & 0.205 & 0.227 \\
\hline cc-pVDZ & 0.733 & 0.803 & 9.5 & 0.034 & 0.083 & 0.205 & 0.221 \\
\hline cc-pVTZ & 0.770 & 0.802 & 4.2 & 0.061 & 0.082 & 0.213 & 0.223 \\
\hline cc-pVQZ & 0.793 & 0.802 & 1.1 & 0.072 & 0.083 & 0.219 & 0.221 \\
\hline cc-pV5Z & 0.797 & 0.802 & 0.6 & 0.076 & 0.084 & 0.219 & 0.221 \\
\hline cc-pV6Z & 0.799 & 0.802 & 0.4 & 0.080 & 0.084 & 0.219 & 0.220 \\
\hline
\end{tabular}

The STO-6G results are for optimised Slater exponents of 1.170 for VB-local and 1.201 for VB-delocal a $\%$ enhancement 
of the molecule. They are not intended to keep the electron density local. This function is a single Gaussian, with exponent $\alpha=0.727$. This value is most appropriate for a hydrogen atom bonded to a heavier element, not another hydrogen atom. For RHF, GVB and G-Weinbaum, the optimum values are $\alpha=1.065, \alpha=1.166$ and $\alpha=1.163$, respectively, indicating that the orbital shrinks. In contrast, for G-Wang the optimum exponent decreases to $\alpha=0.608$, indicating that the orbital expands, as it is reaching out to the other atom to match GVB.

To further investigate the ability of basis functions to modify an orbital far from the point where individual basis functions are centred, the use of a single basis set at the midpoint of the bond was investigated to form the GVB orbitals. Clearly the basis set must include asymmetric functions such as $p, f$ to form two different GVB orbitals. The basis set selected was the $\mathrm{H}$ atom cc-pVnZ basis at the midpoint and not at the atoms. Both cc-pVDZ and cc-pVTZ give orbitals that are very different from the GVB orbitals, and the energy is very high. However, from cc-pVQZ to cc-pV6Z the orbital has the same features as the GVB orbital. For cc-pV6Z, the function along the line of the two atoms is indistinguishable from the GVB function except for close to each nucleus. The energies are poor, due to the inability to easily match the cusp at the nucleus. However, from cc-pVDZ to cc-pV6Z the energy with basis functions centred at the midpoint goes from -0.9942 to $-1.1458 E_{\mathrm{h}}$, close to the cc-pVDZ GVB energy of $-1.1469 E_{\mathrm{h}}$.

Further insight can be gained by removing the $p$ basis function from the cc-pVDZ basis. This gives an overlap of 0.688 for the G-Wang orbitals [Table 2; cf. 0.733 (Table 1)]. Thus the overlap enhancement due to the $\mathrm{p}$ function is quite significant. The extent of the orbital is slightly reduced (Table 2), and the centroid of charge is of course now at the nucleus. VB-local orbitals are thus overlap enhanced orbitals, even with the addition of a single $p$ polarisation function. However, in spite of the small weight of the $p$ function, there is a significant increase in energy, $-1.13645 E_{\mathrm{h}}$ compared to $-1.14289 E_{\mathrm{h}}$ when the standard $\mathrm{p}$ function is excluded.

If all polarisation functions are removed from the cc$\mathrm{pVnZ}$, leaving only $n s$-type basis functions, the G-Wang energy converges fairly rapidly, and the GVB energy converges slightly less rapidly. This is shown in Fig. 1 relative to the results when the polarisation functions are included as discussed earlier. The overlap is essentially constant as $\mathrm{n}$ increases for both G-Wang and GVB functions (see Table 2). The overlap between the GVB orbitals is larger than that of the G-Wang orbitals. With the full basis set, the overlap enhancement is entirely due to the presence of polarisation functions and is already present even when only a single $p$ polarisation function is added.
Table 2 Comparison of the overlap between the two VBOs and the orbital extents (in $\AA^{2}$ ) in $\mathrm{H}_{2}$ using the G-Wang (VB-local) and GVB (VB-delocal) methods using only the $s$-type basis functions from the cc-pVnZ basis sets (+STO-6G for comparison)

\begin{tabular}{llllll}
\hline Basis & \multicolumn{2}{l}{ Overlap } & & \multicolumn{2}{l}{ Extent $\left\langle Z^{2}\right\rangle$} \\
\cline { 2 - 3 } \cline { 5 - 5 } & s-only G-Wang & s-only GVB & & s-only G-Wang s-only GVB \\
\hline STO-6G & 0.687 & 0.797 & & 0.205 & 0.227 \\
cc-pVDZ & 0.688 & 0.797 & & 0.203 & 0.225 \\
cc-pVTZ & 0.687 & 0.794 & & 0.205 & 0.229 \\
cc-pVQZ & 0.686 & 0.793 & & 0.206 & 0.228 \\
cc-pV5Z & 0.686 & 0.793 & & 0.206 & 0.228 \\
cc-pV6Z & 0.686 & 0.793 & 0.205 & 0.228 \\
\hline
\end{tabular}

The G-Weinbaum function uses the VB-local approach, but what happens if the VB-delocal approach is used? Resonance between the GVB function and ionic structures with the same VB orbitals is arbitrary for all basis sets as the two types of structures have zero interaction-the ionic structures are single excitations from the GVB functionsan extended Brillouin's theorem [39]. At the CBS limit, the G-Wang function is identical to the GVB function, so the ionic terms will also have zero interaction with the G-Wang function. Hence, the ionic-covalent ratio becomes undefined at the CBS limit, in much the same way that Mulliken charges do. This is a serious weakness for the idea of ioniccovalent resonance for homonuclear diatomics.

While it is not the purpose of this study to obtain the CBS limit energy, it is interesting to see how close the correlation consistent basis sets can get. The complete basis set limit for the GVB function has been closely approached by Glushkov and Wilson [40, 41] using a large fully optimised basis set of s-type Gaussian functions distributed on a grid starting from even-tempered exponents and an harmonic model for the basis set positions. Their basis functions are distributed over the whole space and are not atom-centred, reminding us that basis functions do not have to be atomcentred to approximate the 1-electron functions in VB theory and that they are indeed just a tool to get the 1-electron functions, balancing accuracy and cost. They used a bond length of $1.4 a_{0}$ (slightly different from the value of $0.74 \AA$ used here) and obtain an energy of $-1.152160 E_{\mathrm{h}}$. For the GVB function in GAMESS(US), but not for VB functions in VB2000 and GAMESS-UK, we can use $\mathrm{h}$ basis functions in the cc-pV6Z and aug-cc-pV6Z basis sets, leading to acceptable extropolations to the CBS limit. This is not possible for the G-Wang function. Using the cc-pV6Z and aug-cc-pV6Z basis sets and Cartesian basis functions for $\mathrm{H}_{2}$ at $1.4 a_{0}$, energies are found that are $2.9 \times 10^{-6} E_{\mathrm{h}}$ and $2.2 \times 10^{-7} E_{\mathrm{h}}$ higher, respectively. Convergence of the energy to the CBS limit is always slow. Jensen [42] 
investigated the Hartree-Fock CBS limit for $\mathrm{H}_{2}$ in detail. This allows us to conclude that the exponential formula [43] for obtaining the CBS limit is better than the power formula [44]. For the GVB limit, there is little direct experience of which method to use. The aug-cc-pp-V6Z basis gives an estimate for the CBS limit at $0.74 \AA$ of -1.152168 $E_{\mathrm{h}}$ using the exponential formula to fit the HF energy and the correlation energy separately. Using the energy difference with the aug-cc-pp-V6Z basis, this suggests a CBS limit at $1.4 a_{0}$ of $-1.152184 E_{\mathrm{h}}$, about $2.0 \times 10^{-6} E_{\mathrm{h}}$ below Wilson's estimate.

This convergence of VB-local and VB-delocal has led some valence bond groups to limit the basis sets with the VB-local approach to rather small basis sets in order to keep the VB orbitals closer to the local nature that they deem to be essential for describing classical VB structures [45]. This is controversial, with other groups suggesting that the VB-delocal orbitals are localised enough to match the classical structures, even if they are slightly distorted [46]. It is clear from this work on the hydrogen molecule that the addition of any polarisation function enhances the overlap and displaces the centroid of charge from the nucleus and distorts the VBO into the bond. These orbitals cannot be considered to be "strictly localised". The use of "strictly" in this context should be discouraged. With a small number of polarisation functions, they are certainly more localised than the VB-delocal orbitals, but the term "strictly localised" should be restricted to basis sets with no polarisation functions using the VB-local approach. This is the s-only G-Wang method shown in Fig. 1. It converges well to a limit, but this s-only limit is well above the CBS limit. It has also been reported that this is close to the OPTX density functional method for distances close to the equilibrium distance with similar amounts of both dynamic correlation and left-right correlation [47, 48]. The VB-delocal approach with s-only basis sets lowers the energy, but its limit is higher than that for the full G-Wang VB-local. It has no advantages over other methods.

The orbitals for the G-Wang and GVB VB-delocal methods are compared in Fig. 2 for three different basis sets. Only the very largest basis sets begin to show the cusp correctly at both nuclei. Small basis sets give differences over most of the range. For cc-pVQZ and above, the differences are large only close to the two nuclei. But a natural question is whether the cusp at the second nucleus is actually correct. If the best form of the VB orbital is obtained, there will be a response near both nuclei to the discontinuity in the potential, leading to cusps. A cusp will appear for VBlocal expansions of the VB orbitals in G-Wang if a sufficiently large basis set including very high angular momentum functions is used. The application of orbital restrictions to maintain the orbitals local is equivalent to a limitation of the 1-electron basis set, and thus the VB-local expansions do not use the full flexibility of the basis.

\subsection{CASSCF(2,4)-related methods}

The use of four orbitals rather than two orbitals allows the introduction of different orbitals in the covalent and ionic terms of the G-Weinbaum method. The G-Weinbaum function of Eq. 5 is replaced by:

$$
\begin{aligned}
\psi= & \left\{\left[\phi_{\mathrm{A} 1}(1) \phi_{\mathrm{B} 1}(2)+\phi_{\mathrm{B} 1}(1) \phi_{\mathrm{A} 1}(2)\right]\right. \\
& \left.+\lambda\left[\phi_{\mathrm{A} 2}(1) \phi_{\mathrm{A} 2}(2)+\phi_{\mathrm{B} 2}(1) \phi_{\mathrm{B} 2}(2)\right]\right\}[\alpha(1) \beta(2)-\beta(1) \alpha(2)]
\end{aligned}
$$

with $\phi_{\mathrm{A} 1}$ and $\phi_{\mathrm{A} 2}$ centred on atom $\mathrm{A}$ and $\phi_{\mathrm{B} 1}$ and $\phi_{\mathrm{B} 2}$ on atom $\mathrm{B}$.

This is an example of the breathing orbital approach [25-28]. The four molecular orbitals used in the CAS$\operatorname{SCF}(2,4)$ are the $1 s$ and $2 s$ bonding and antibonding combinations. For the breathing orbital functions, the VB-local approach is used. Energies are plotted in Fig. 3, where the energies for all methods from the G-Wang to
Fig. 2 a G-Wang local orbitals for different basis sets, and $\mathbf{b}$ GVB delocal orbitals for different basis sets

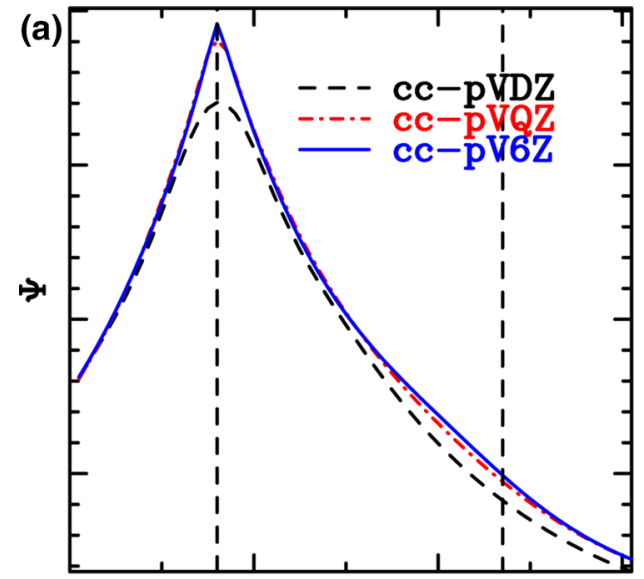

R

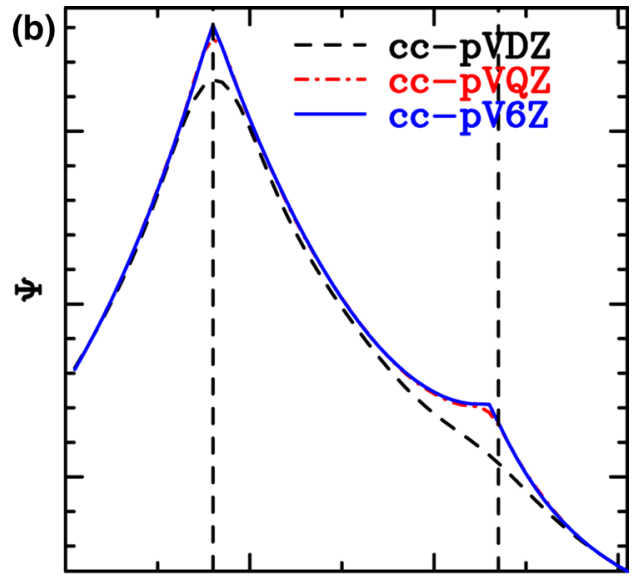

$\mathbf{R}$ 


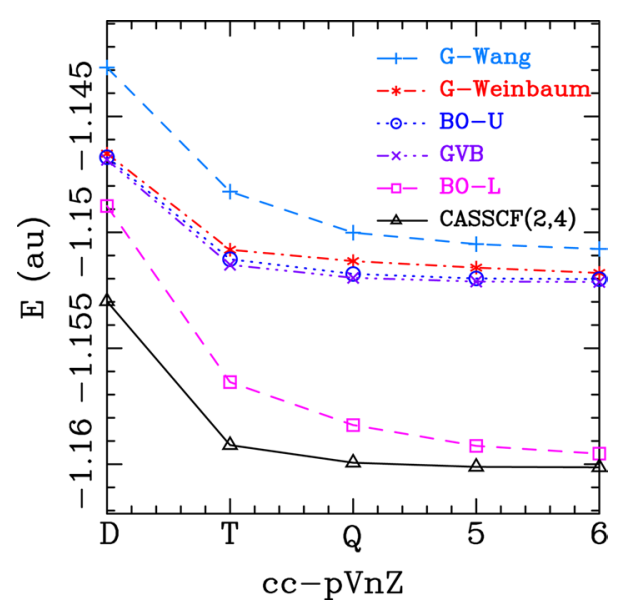

Fig. 3 G-Wang, G-Weinbaum, BO-U, GVB, BO-L and CAS$\operatorname{SCF}(2,4)$ energies as a function of basis set

$\operatorname{CASSCF}(2,4)$ are shown. An interesting feature arises. There appears to be two breathing orbital wavefunctions. The first, designated BO-U (breathing orbital upper), differs only slightly from those in the G-Weinbaum wavefunction. The energy is also only lowered slightly, and it still lies above the $\operatorname{GVB} / \operatorname{CASSCF}(2,2)$ energy. This breathing orbital function is still part of the CAS$\operatorname{SCF}(2,2)$ family of methods. This breathing orbital function is the one generated by the XMVB program with the recommended choice of initial orbitals from the G-Weinbaum function. If VB-delocal orbitals are used for BO-U, the function would be identical to GVB. The second wavefunction, designated BO-L, gives VB orbitals for the ionic structure that are much more diffuse than those for the covalent structure. They contain a node and look like $2 s$ functions. This is not unexpected as CAS$\operatorname{SCF}(2,4)$ includes $2 s$ MOs. This breathing orbital function with VB-local is clearly part of the $\operatorname{CASSCF}(2,4)$ family of methods. When VB-delocal orbitals are used for BO-L, the energy is so close to that of $\operatorname{CASSCF}(2,4)$ that it cannot be distinguished when plotted. However, it appears that it does not include some very minor contributing structures to $\operatorname{CASSCF}(2,4)$. BO-L will have this same CBS limit.

\subsection{CASSCF (2,6)- and CASSCF (2,8)-related methods}

If the s-like orbitals $\left[\phi_{\mathrm{A} 2}(1) \phi_{\mathrm{A} 2}(2)+\phi_{\mathrm{B} 2}(1) \phi_{\mathrm{B} 2}(2)\right]$ in Eq. 7 are replaced by $p$-like orbitals giving $\left[p_{\mathrm{Ax}}(1) p_{\mathrm{Ax}}(2)+\right.$ $\left.p_{\mathrm{Bx}}(1) p_{\mathrm{Bx}}(2)+p_{\mathrm{Ay}}(1) p_{\mathrm{Ay}}(2)+\mathrm{p}_{\mathrm{By}}(1) p_{\mathrm{By}}(2)\right]$, where $\mathrm{p}_{\mathrm{Ax}}$ and $p_{\mathrm{Bx}}$ are $p_{x}$ orbitals centred on atoms $\mathrm{A}$ and $\mathrm{B}$, and $p_{\mathrm{Ay}}$ and $p_{\mathrm{By}}$ are $p_{\mathrm{y}}$ orbitals on atoms $\mathrm{A}$ and $\mathrm{B}$, they add a different kind of correlation. The $x$ axis and $y$ axis are perpendicular to the molecular axis. This belongs to the $\operatorname{CASSCF}(2,6)$ family of methods where the 6 MOs are the $\sigma_{1 s}$ bonding and antibonding MOs and the $p_{x}$ and $p_{y}$ $\pi$ bonding and antibonding MOs. This has a lower energy than CASSCF $(2,4)$. This method was used by Wilson and Gerratt [49] who named it the generalised HirschfelderLinnett method as it replaced single atomic orbitals in the original Hirschfelder-Linnett method by optimised VBOs [50]. We designate this method as GHL-1. However, for consistency, we use VB-local orbitals, while Wilson and Gerratt use VB-delocal orbitals. If the above VB wavefunction is solved with VB-delocal orbitals, the energy is so close to that of $\operatorname{CASSCF}(2,6)$ that it cannot be distinguished when plotted. However, it appears that it does not include some very minor contributing structures to $\operatorname{CASSCF}(2,6)$.

If the four $p$-based structures are added to the $s$-based breathing orbital structures, rather than replacing them, a function in the $\operatorname{CASSCF}(2,8)$ family of methods is obtained. This, a further generalisation of the Hirschfelder-Linnett method is designated as GHL-2. Again the VB-local approach is used. When VB-delocal orbitals are used for GHL-2, the energy is indistinguishable when plotted from the $\operatorname{CASSCF}(2,8)$, and again, minor contributing structures are missing from GHL-2 compared to $\operatorname{CASSCF}(2,8)$.

Results for these methods are shown in Fig. 4. Also shown in Fig. 4 is the energy of the full CI method, which for the hydrogen molecule is identical to configuration interaction and coupled cluster with all single and double excitations-CISD and CCSD. The MP2 energy lies just above the GHL-2 energy, and $\operatorname{CASSCF}(2,8)$ is close to full CI. The VB-local energies are converging to the same limit as their VB-delocal counterparts as the functions are formally identical.

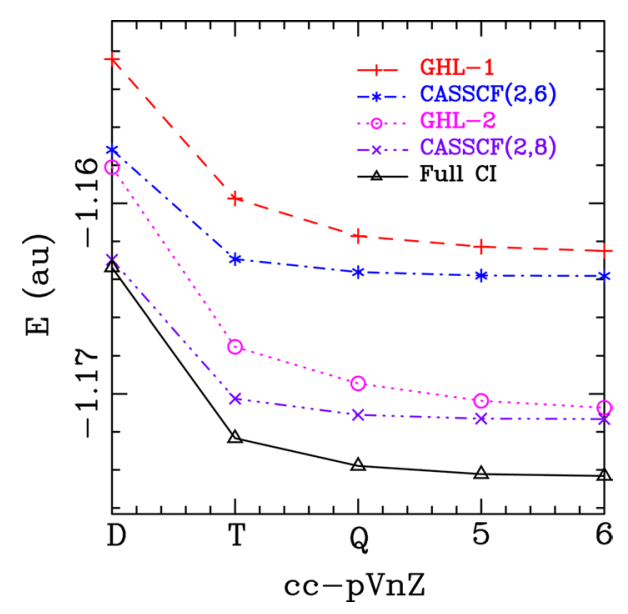

Fig. 4 GHL-1, $\operatorname{CASSCF}(2,6)$, GHL-2, $\operatorname{CASSCF}(2,8)$, and full CI energies as a function of basis set 


\section{Conclusion}

The simplest picture of bond formation is that as two hydrogen atoms approach, they overlap and couple exactly as Heitler and London told us back in 1927. However, the VB orbitals do not remain as unaltered free atom functions but contract and distort to give the best VB orbitals for the molecule itself. As we see here, they remain associated in turn with the two atoms and in that sense they are localised.

Plotting the "VB-local" and "VB-delocal" energies highlights that VB functions are in families related to particular levels of CASSCF. If two VB functions that are formally identical but have 1-electron orbitals that belong to different sets of basis sets that can converge to the complete basis set (CBS) limit, it is clear that the two basis set limits are identical. This is the case where the two VB functions differ in one using the "VB-local" approach and one using the "VB-delocal" approach. They have the same CBS limit.

For the CASSCF $(2,2)$ case, GVB is identical to CAS$\operatorname{SCF}(2,2)$. GVB and G-Wang bracket the covalent-ionic resonance G-Weinbaum energy. The equality of the "VB-local" and "VB-delocal" approach CBS limits is not disputed, but it is sometimes suggested that the CBS limit is so difficult to achieve that they really are different. If the cc-pVnZ basis is used, or better the aug-cc-pVnZ, particularly if the molecule has regions that are negatively charged, then the CBS limit with the "VB-delocal" approach is as easy to obtain as it is with the Hartree-Fock or coupled cluster methods.

Both the overlap between the VBOs converges and the centroids of charge converge for "VB-local" and "VBdelocal" to the same value. While "VB-local" and "VBdelocal" are very different with a minimum basis set, it is remarkable how "VB-local" catches up when polarisation functions are added. The "VB-local" orbitals with the ccpV6Z basis set are essentially just as distorted towards the other atom as are the "VB-delocal" orbitals. Similarly the extent of the orbital in the "VB-local" case is converging well to the "VB-delocal" result.

Convergence of the energy to the CBS limit is slower. If the energy lowering from the G-Wang/cc-pVDZ energy to the best estimate of the CBS limit is used for comparison, then the "VB-local" approach with cc-pV6Z has achieved a $84.4 \%$ lowering compared with a $99.7 \%$ lowering for the "VB-delocal" approach. The "VB-local" lowering of $84.4 \%$ is not insignificant. The reason for the slower convergence of the "VB-local" approach is failure to match the "VB-delocal" approach close to the second nucleus. Nevertheless, the "VB-local" with larger basis sets is beginning to match to the changes in the function at the second nucleus. More significantly, there is no clear point when the orbitals cease to be "strictly localised" and become delocalised. As soon as polarisation functions are included in the basis set, the "VB-local" orbitals start to move out towards the neighbour atom.

One advantage of the "VB-delocal" approach over the "VB-local" approach is that it is not restricted to atom-centred basis sets. The GVB orbitals built from basis functions centred on the bond midpoint give good agreement with the cc-pV6Z results from the atom-centred "VB-local" orbitals and "VB-delocal" orbitals for overlap (0.802), position of centroid of charge ( 0.0846 from a $\mathrm{H}$ atom) and extent $\left(\left\langle Z^{2}\right\rangle=0.2186\right)$.

Two breathing orbital function solutions have been observed. Choosing an inner basis function as the initial guess for the VBOs leads to the BO-U solution, while choosing an outer basis function leads to the BO-L solution. A second lower energy breathing orbital function may be important in other systems, but will not be found using the standard XMVB guess of the G-Weinbaum orbitals for breathing orbitals.

Going beyond the use of two orbitals leads to significant lowering of the energy while retaining a simple VB picture. This is particularly so by adding the $p$ functions perpendicular to the molecular axis. If VB-delocal orbitals are used for the GHL-1 method, the energy is indistinguishable from $\operatorname{CASSCF}(2,6)$. Adding the $2 s$-like functions as well gives a VB picture indistinguishable from $\operatorname{CASSCF}(2,8)$, which is close to full CI. In both cases, the VB-local approach is converging well to the VB-delocal limit.

Increasing the basis set towards the CBS limit clearly shows that while the energy converges slowly due to difficulties with Gaussian functions to fit VBOs close to both nuclei, the general form of the VBOs converges much faster as shown by the enhancement of the overlap, the movement of the centroid of charge from the nucleus and the extent of the orbitals. The VB orbitals cannot remain strictly localised if polarisation functions are included. This supports the general use of VB-delocal orbitals over VBlocal orbitals. The difference between these approaches blurs as the basis set is increased. A further advantage of the VB-delocal orbital approach is it leaves open the possibility that the final orbitals will not be like atomic orbitals. Experience with the SC method shows that this can occur but is not common. The VB-delocal orbitals reported here are only slightly distorted atomic orbitals thus retaining the valence bond view of bonding due to overlap between atom-based orbitals.

Acknowledgments B.J.D. acknowledges the collaboration with Dr. Jiabo Li on the VB2000 program and Dr. R. Harcourt for valuable discussions. R.W.A.H. acknowledges Prof. R. Broer (University of Groningen, The Netherlands) for fruitful discussions and the Zernike Institute for Advanced Materials ("Dieptestrategie" program) for financial support. The work was partially done with computing time at the Dutch National Supercomputer Cartesius (SURFsara, SH-21313) and the Monash Sun Grid at Monash University. 
Open Access This article is distributed under the terms of the Creative Commons Attribution 4.0 International License (http://creativecommons.org/licenses/by/4.0/), which permits unrestricted use, distribution, and reproduction in any medium, provided you give appropriate credit to the original author(s) and the source, provide a link to the Creative Commons license, and indicate if changes were made.

\section{References}

1. Heitler W, London F (1927) Z Phys 44:455-472

2. McLean AD, Weiss A, Yoshimine M (1960) Rev Mod Phys 12:211-218

3. Szabo A, Ostlund NS (1982) Modern quantum chemistry. MacMillan Publishing, New York

4. Laaksonen L, Pyykko P, Sundholm D (1983) Int J Quantum Chem 23:319-323

5. Cramer CJ (2004) Essentials of computational chemistry, 2nd edn. Wiley, London, pp 227-230

6. Schwenke DW (2012) Mol Phys 110:2557-2567

7. Velilla L, Paniagua M, Aguado A (2011) Int J Quantum Chem 111:387-399

8. Bickelhaupt FM, Baerends EJ (2000) Kohn-Sham density functional theory: predicting and understanding chemistry. In: Lipkowitz KB, Boyd DB (eds) Reviews in computational chemistry, vol 15. Wiley-VCH, Weinheim, pp 1-86

9. Thorsteinsson T, Cooper DL, Gerratt J, Karadakov PB, Raimondi M (1996) Theor Chim Acta 93:343-366

10. Gerratt J (1971) Adv Atom Mol Phys 7:141-221

11. Gerratt J (1971) General theory of spin-coupled wavefunctions for atoms and molecules. In: Bates D, Esterman I (eds) Advances in atomic and molecular physics, vol 7. Academic Press, New York, pp 141-221

12. Gerratt J, Raimondi M (1980) Proc R Soc Lond A 371:525-552

13. Cooper DL, Gerratt J, Raimondi M (1984) Faraday Symp Chem Soc 19:149-163

14. Cooper DL, Gerratt J, Raimondi M (1987) Modern valence bond theory. In: Lawley KP (ed) Advances in chemical physics: ab initio methods in quantum chemistry part 2 , vol 69 . Wiley, London, pp 319-397

15. Wang SC (1928) Phys Rev 31:579-586

16. Mo Y, Hiberty PC, Schleyer PvR (2010) Theor Chem Acc 127:27-38

17. Rashid Z, van Lenthe JH, Havenith RWA (2012) J Phys Chem A 116:4778-4788

18. McWeeny R (1988) Theor Chim Acta 73:115-122

19. McWeeny R (1988) Int J Quantum Chem 34:25-36

20. Mo Y, Lin Z, Wu W, Zhang Q (1996) J Phys Chem 100:11569-11572

21. Weinbaum S (1933) J Chem Phys 1:593-596
22. Coulson CA, Fischer I (1949) Philos Mag 40:386-393

23. Goddard WA III, Dunning TH Jr, Hunt WJ, Hay PJ (1973) Acc Chem Res 6:368-376

24. Goddard WA III, Harding LB (1978) Ann Rev Phys Chem 29:363-396

25. Hiberty PC (1997) J Mol Struct (Theochem) 398-399:35-43

26. Hiberty PC, Flament JP, Noizet E (1992) Chem Phys Lett 189:259-265

27. Hiberty PC, Humbel S, Byrman CP, van Lenthe JH (1994) J Chem Phys 101:5969-5976

28. Hiberty PC, Shaik S (2002) Theor Chem Acc 108:255-272

29. Dunning TH Jr (1989) J Chem Phys 90:1007-1023

30. Woon D, Dunning TH Jr (1993) J Chem Phys 98:1358-1371

31. Verbeek J, Langenberg JH, Byrman CP, Dijkstra F, Engelberts JJ, Zielinski M, van Lenthe JH (1988-2015) TURTLE, an ab initio VB/VBSCF program. Utrecht, The Netherlands

32. Guest MF, Bush IJ, van Dam HJJ, Sherwood P, Thomas JMH, van Lenthe JH, Havenith RWA, Kendrick J (2005) Mol Phys 103:719-747

33. Li J, Duke BJ, McWeeny R (2013) VB2000 version 2.7, October 2013. SciNet Technologies, San Diego

34. Li J, McWeeny R (2002) Int J Quantum Chem 89:208-216

35. Gordon MS, Schmidt MW (2005) Advances in electronic structure theory: GAMESS a decade later. In: Dykstra CE, Frenking G, Kim KS, Scuseria GE (eds) Theory and applications of computational chemistry: the first forty years. Elsevier, Amsterdam, pp 1167-1189

36. Schmidt MW, Baldridge KK, Boatz JA, Elbert ST, Gordon MS, Jensen JH, Koseki S, Matsunaga N, Nguyen KA, Su S, Windus TL, Dupuis M, Montgomery JA (1993) J Comput Chem 14:1347-1363

37. Song L, Chen Z, Ying F, Song J, Chen X, Su P, Mo Y, Zhang Q, Wu W (2012) XMVB 2.0: an ab initio non-orthogonal Valence Bond Program. Xiamen University, Xiamen

38. Song L, Mo Y, Zhang Q, Wu W (2005) J Comput Chem 26:514-521

39. Levy B, Berthier G (1968) Int J Quantum Chem 2:307-319

40. Glushkov VN, Wilson S (2009) Mol Phys 107:2299-2308

41. Glushkov VN, Wilson S (2012) Mol Phys 110:149-161

42. Jensen F (1999) J Chem Phys 110:6601-6605

43. Helgaker T, Klopper W, Koch H, Noga J (1997) J Chem Phys 106:9639-9646

44. Halkier A, Helgaker T, Jørgensen P, Klopper W, Koch H, Olsen J, Wilson AK (1998) Chem Phys Lett 286:243-252

45. Hiberty PC (2007) Faraday Discuss 135:367-401

46. Cooper DL, Gerratt J, Raimondi M (1986) Nature 323:699-701

47. Handy NC (2009) Mol Phys 107:721-726

48. Peach MJG, Tozer DJ, Handy NC (2011) Int J Quantum Chem 111:563-569

49. Wilson S, Gerratt J (1975) Mol Phys 30:777-787

50. Hirschfelder J, Linnett J (1950) J Chem Phys 18:130-142 